\section{Exercise interventions for juvenile fibromyalgia: current state and recent advancements}
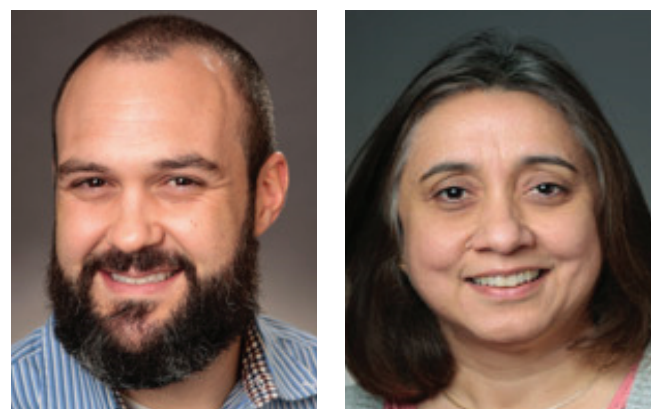

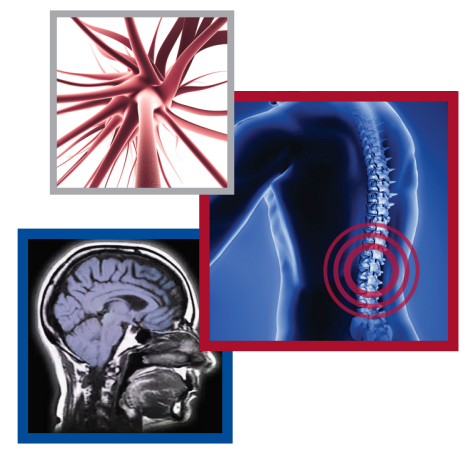

William R Black \& Susmita Kashikar-Zuck* ${ }^{* 1,2}$

First draft submitted: 21 December 2016; Accepted for publication: 18 January 2017; Published online: 3 February 2017

Juvenile fibromyalgia (JFM) is a chronic pain condition affecting between 2.1 and $6.1 \%$ of school-aged children [1] and characterized by widespread musculoskeletal pain, fatigue, sleep difficulties and impairment in physical functioning [2]. Adolescents with JFM tend to be very sedentary, despite treatment recommendations that encourage physical exercise and greater participation in physical activity [3]. Along with greater sedentary behavior, evidence of deconditioning, reduction in strength and altered movement biomechanics have been documented in adolescents with JFM accompanied by self-reported fear of movement [4]. These findings highlight the need for a more comprehensive approach to physical exercise interventions in this population. In this article, we first review the evidence for the beneficial effects of a variety of physical exercise approaches in fibromyalgia (FM). We then discuss findings from studies that provide clues as to why patients with FM find exercise particularly challenging. And finally, we describe recent studies evaluating a new integrative intervention that may help adolescents with JFM engage in physical exercise with greater ease and confidence.

What is the evidence behind exercise recommendations for JFM?

The American Pain Society guidelines suggest that individuals with FM engage in moderate to vigorous physical exercise for at least $30 \mathrm{~min}$ two- to three-times per week. Evidence for the benefits of exercise are primarily based on treatment studies in adults with FM, which have focused on strength/resistance training, aerobic exercise, or movement-based therapies such as yoga, tai chi, among others [5]. These studies have generally shown positive effects for exercise on pain reduction and improved daily functioning. Specifically, resistance exercise appears to be associated with reduction in physical fatigue [6], while aerobic exercises (e.g., walking, stationary cycling

\section{KEYWORDS}

- juvenile fibromyalgia

- neuromuscular training • physical exercise

\section{"Juvenile fibromyalgia is a chronic pain condition affecting between 2.1 and $6.1 \%$ of school-aged children."}


"Functional MRI studies have shown that regular participation exercise is associated with beneficial changes in pain perception in fibromyalgia patients at a neurological level via the stimulation of brain regions

in the descending pain inhibition pathway, improving pain modulatory capabilities.” and aquatic exercise) seem to have a stronger effect on pain reduction [7]. With regards to JFM, there has been only one small-scale trial of aerobic exercise for adolescents with JFM. In this study, Stephens et al. evaluated the feasibility and effectiveness of a 12-week program, in which participants were randomized to either an aerobics training program or qigong (i.e., the control condition) [8]. Both groups experienced improvement in FM-related treatment outcomes, but participants in the aerobic exercise condition exhibited greater improvements in fatigue, quality of life and daily functioning. Despite the known benefits of exercise, one of the main concerns related to traditional exercise programs is that of poor long-term adherence to exercise in patients with FM [9]. It has been proposed that less strenuous forms of physical activity that are more self paced may lead to greater engagement and adherence to physical activity [10]. For example, a lifestyle physical activity approach consists of strategies to reduce sedentary behavior and to increase movement throughout the day, such as standing to complete activities, taking regular walking and stretch breaks throughout the day, and increasing incidental exercise. Incorporating such activities into a daily routine may help individuals develop lifestyle modifications that are more sustainable in the long term. It is currently not known whether such modifications are associated with better long-term adherence. Finally, studies of movement-based complementary therapies, such as yoga, Tai Chi and Qigong have shown beneficial effects on pain reduction in chronic musculoskeletal pain [5]. Practiced at lower intensities, these activities are not based on aerobic fitness, but rather incorporate physical exercise with deep breathing and mindfulness which are complementary techniques also known to help in pain management. Taken together, these findings suggest that traditional methods of physical exercise such as aerobic and resistance training may be challenging for many patients with FM but that starting with less intense forms of exercise and incorporating other mind-body approaches may be useful to consider for such patients.

\section{What do we know about the underlying neurobiology of how exercise impacts pain?}

Recent advancements in neuroscience research have pointed to some of the reasons why exercise may be more painful or unpleasant for patients with chronic pain, especially those with widespread (sometimes referred to as 'central sensitization') pain syndromes such as FM. For healthy individuals, acute bouts of exercise tend to reduce pain sensitivity (i.e., create hypoalgesia) through the release of endogenous opioids, which have been proposed to help modulate pain processing in areas of the brain and spinal cord. Therefore, exercise is generally expected to result in higher pain tolerance and reduced pain [11]. However, in patients with chronic pain, acute aerobic and isometric exercise may actually have the opposite (hyperalgesic) effect; in other words, pain sensitivity may increase immediately following exercise [12]. Patients with chronic pain are known to have neurobiological alterations characterized by central sensitization, as well as deficits in descending pain modulation which may play a role in this hypersensitivity [13]. These underlying neurobiological differences may explain why exercise tends to be immediately reinforcing for healthy individuals, whereas in patients with FM, acute exercise may be associated with increased pain and discomfort. An association of heightened pain with exercise may have an impact on an individual's willingness to persevere with prescribed exercise, contributing to persistent fear and avoidance of activity. This is troubling for pain researchers and clinicians alike, as regular exercise and physical activity have been shown to reduce chronic pain by intervening on the very neurobiological pathways that are associated with acute increases in pain sensitivity.

Functional MRI studies have shown that regular participation exercise is associated with beneficial changes in pain perception in FM patients at a neurological level via the stimulation of brain regions in the descending pain inhibition pathway, improving pain modulatory capabilities [14]. Together, these findings suggest that neurobiological changes associated with regular exercise have the potential to lead to long-term pain reduction, if patients can overcome the short-term unpleasant increases in pain after exercise and avoid the increased fear of movement which further discourages patients with FM pain from engaging in exercise and physical activity [15].

\section{What is the importance of movement} biomechanics in exercise?

Fear of engaging in physical activity may not only be influenced by pain associated with the 
neurobiological alterations mentioned above but also due to changes in biomechanics (or how people move) when they are in constant pain. Clinicians have long observed that patients with chronic pain demonstrate changes in their gait and posture, showing more guarded movements and favoring the body area in which they have pain. Recent advances in technology including 3D motion capture used by specialized human performance laboratories (e.g., in the field of sports medicine and injury prevention) have made it possible to apply these technologies to analyze gait, posture, balance and movement biomechanics in patients with chronic pain. In a study of adolescents with JFM compared with same-age healthy peers, our research group showed that adolescents with JFM did not show any differences from their peers in walking gait, but showed deficits in movement biomechanics as tasks become even slightly more challenging (e.g., jumping off a $30 \mathrm{~cm}$ box). These deficits were similar to those associated with risk for injury in studies of adolescent athletes [16] and may similarly place patients with JFM at greater risk for pain associated with poor biomechanics. Although studies in this area are still in the early stages, it is reasonable to speculate that these deficits in biomechanics should be addressed before adolescents with JFM begin to engage in more strenuous types of aerobic or resistance exercise to prevent injury and reduce the likelihood of pain flares.

\section{Development of a new exercise approach for adolescents with JFM}

Given what we are learning about the neurobiological responses to exercise in individuals with chronic pain and the findings of altered biomechanics that may explain why patients with JFM might find physical exercise more challenging, new approaches to facilitate exercise are being considered. Tailored and progressive exercise programs focused on improving core strength and biomechanics that facilitate movement competence and designed to minimize muscle soreness when initiating new exercises, could be particularly well suited for JFM.

\section{- Neuromuscular training}

Neuromuscular training (NMT), drawn from the field of pediatric exercise science and sports medicine, is emerging as a particularly promising approach to address biomechanical deficits in JFM [4]. NMT has been previously applied to reduce sports-related injuries in children and adolescents [16]. Exercises in NMT programs focus on improving posture, gait, balance, strength and fundamental movement by progressing the participants through various isometric, concentric, eccentric and functional exercises, incorporating both resistance training and dynamic stability that are low impact and technique oriented. Exercises at each progression can be modified according to the individual's ability level and specific motor control deficits, and individuals do not progress to successive levels until sufficient movement competence is developed. Through such a program that focuses on improving movement mechanics along this progression, both injury risk and delayed onset muscle soreness can be minimized, potentially improving treatment acceptability and adherence in JFM. A full protocol for NMT that has been adapted for adolescents with JFM has been previously published [17] and one example of the specific exercise progression from isometric, concentric and eccentric phases to full functional movement (double leg deep hold) is presented in Table 1. Early results show that NMT can help adolescents with JFM increase their self-efficacy in their ability to engage in exercise through increased movement competence and decreased fear about engaging in exercise - and it is anticipated that this will ultimately increase their engagement in more vigorous physical activity.

\section{- Incorporating cognitive-behavioral approaches with exercise}

Cognitive-behavioral therapy (CBT) is a type of behavioral treatment that has been found to be effective in reducing disability and improving pain coping efficacy in adolescents with JFM [19]. CBT involves training in adaptive coping skills such as activity pacing, calming strategies, distraction, stress management as well as discussion of healthy lifestyle habits. Many of the techniques included in CBT training can be usefully incorporated with NMT, especially given the fear of movement and anxiety about activities that may cause muscle soreness or discomfort in patients with JFM. For example, patients might use breathing relaxation and pacing techniques when they are learning a new exercise that they find challenging. They may also use cognitive techniques, such as self-calming statements, to help them manage their anticipatory anxiety about engaging in unfamiliar exercises. Such strategies would facilitate motivation and

\section{"Tailored and progressive exercise programs focused on improving core strength and biomechanics that facilitate movement competence and designed to minimize muscle soreness when initiating new exercises, could be particularly well suited for juvenile fibromyalgia."}




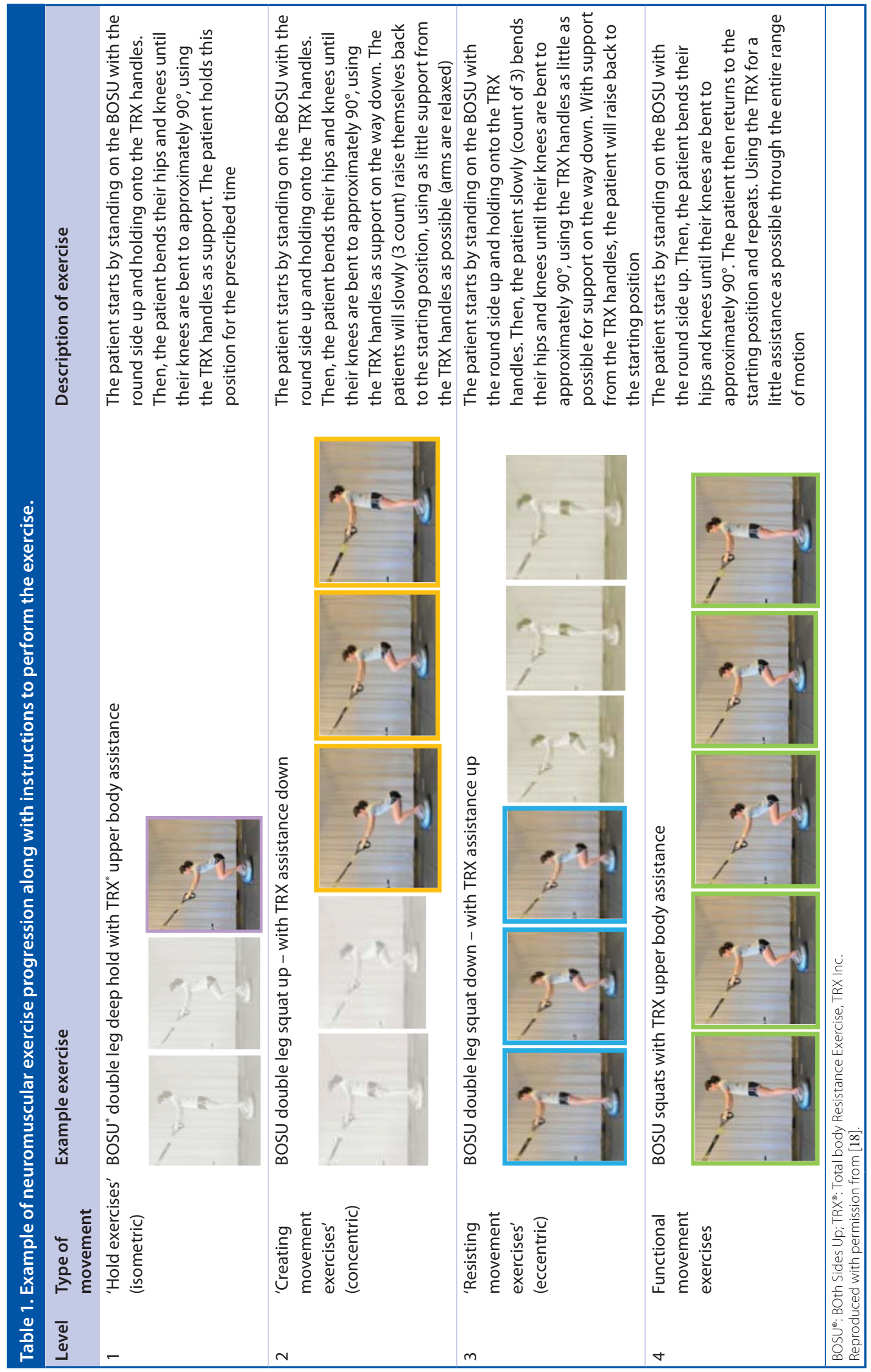


allow patients to realize the full benefits of exercise training through greater engagement and improved adherence.

\section{Integrated programs: combining exercise} \& $C B T$

Both CBT and exercise-based interventions have demonstrated independent effectiveness for JFM outcomes in research studies and combined CBT and physical activity based interventions are often used clinically as part of multidisciplinary pain treatment programs. An intensive day-hospital program integrating physical, occupational and psychological therapies for the treatment of a broad range of chronic pain disorders were found to reduce functional impairment, pain-related fear and increase in readiness to change [20]. Intensive outpatient multidisciplinary programs have also reported improved pain and quality of life in patients with JFM [21]. These programs by and large use traditional aerobic exercise approaches and although the programs can be effective, at least in the short term, long-term adherence to physical activity recommendations tends to remain problematic in patients with complex pain conditions such as JFM; thus, further work is needed to develop exercise programs that correct underlying deficits and are more sustainable.

\section{- FM integrative training for teens}

Our research group has begun to evaluate whether the integration of specialized NMT with CBT in a new Fibromyalgia Integrative Training for Teens program (FIT Teens) for adolescents with JFM [16] can provide a useful alternative to traditional approaches to exercise treatments. A unique aspect of this program is the integration of physical activity programming from the fields of sports medicine and injury prevention that has not typically been applied to patients with chronic pain. Another unique aspect is full integration and real-time coaching by both CBT and physical therapists in the use of psychological coping strategies, as they learn neuromuscular exercises to improve their movement competence. In the FIT Teens program, adolescents participate in a 16 -session, 8 -week group-based program jointly with a CBT therapist and an exercise trainer/physical therapist. Sessions include psychoeducation about pain with CBT-based coping skills training and NMT sessions. Individualized modifications to the exercise component are made for each participant as needed to accommodate for their abilities and baseline fitness.

Through an iterative process of development in which feedback from the patients was incorporated into the design of the program, the FIT Teens protocol was subjected to a rigorous first phase of qualitative evaluation in which it was shown to be feasible and very well tolerated by adolescents with JFM [16]. After completing the program, participants reported feeling as though the program was "more tailored towards people with JFM," and that they believed they would do 'really well' with continuing exercises after the program. They reported feeling stronger, having less fatigue, taking less naps and being more active. With regard to NMT, teens reported that the exercises were initially somewhat challenging but with practice, the exercises got easier [18]. The incorporation of CBT-based skills training was also seen as a plus in helping them deal with challenges of learning new exercises. In a second phase of quantitative testing of the program, JFM patients demonstrated significant reductions in disability, fear of movement and pain catastrophizing, as well as significant improvement in their biomechanical performance (e.g., stride length, hip abduction strength and dynamic postural control). Ongoing studies are testing the benefits of FIT Teens and whether it is more effective than currently used exercise approaches in reducing pain and disability and promoting long-term exercise engagement.

\section{Conclusion \& future perspective}

Having patients participate in regular exercise over the long term has been a particularly challenging issue for pain management providers treating adolescents with JFM. Integrated pain treatment programs, particularly those that incorporate active coping skills training (i.e., CBT approaches) along with physical exercise appear to be beneficial. New research is beginning to incorporate knowledge from fields such as neuroscience and sports medicine to develop more targeted treatments that address the specific deficits in those suffering from chronic pain to make exercise more tolerable and engaging. The integration of NMT approaches from injury prevention/sports medicine may uniquely be able to prepare youth with JFM for regular participation in planned physical exercise, potentially reducing the burden of pain and pain-related disability as they become adults. Further study is needed to determine how 
improved biomechanics and movement competence impact exercise adherence and the neurobiological changes in pain pathways that result from regular physical exercise participation. It will then be possible to achieve increasingly sophisticated and tailored exercise programs for patients with chronic pain conditions such as JFM which will ultimately minimize long-term pain-related disability and suffering for these youth.
Financial \& competing interests disclosure Current work is funded by the National Institute of Arthritis and Musculoskeletal and Skin Diseases of the NIH Grant K24AR056687 to S Kashikar-Zuck. The authors have no other relevant affiliations or financial involvement with any organization or entity with a financial interest in or financial conflict with the subject matter or materials discussed in the manuscript apart from those disclosed.

No writing assistance was utilized in the production of this manuscript.

\section{References}

1 Sardini S, Ghirardini M, Betelemme L, Arpino C, Fatti F, Zanini F. [Epidemiological study of a primary fibromyalgia in pediatric age]. Minerva Pediatr. 48(12), 543-550 (1996).

2 Kashikar-Zuck S, Ting TV. Juvenile fibromyalgia: current status of research and future developments. Nat. Rev. Rheumatol. 10(2), 89-96 (2014).

3 Kashikar-Zuck S, Flowers SR, Strotman D, Sil S, Ting TV, Schikler KN. Physical activity monitoring in adolescents with juvenile fibromyalgia: findings from a clinical trial of cognitive-behavioral therapy. Arthritis Care Res. 65(3), 398-405 (2013).

4 Sil S, Thomas S, Dicesare C et al. Preliminary evidence of altered biomechanics in adolescents with juvenile fibromyalgia. Arthritis Care Res. 67(1), 102-111 (2015).

5 Mist SD, Firestone KA, Jones KD. Complementary and alternative exercise for fibromyalgia: a meta-analysis. J. Pain Res. 6, 247-260 (2013).

6 Ericsson A, Palstam A, Larsson A et al. Resistance exercise improves physical fatigue in women with fibromyalgia: a randomized controlled trial. Arthritis Res. Ther. 18, 176 (2016).

7 Ambrose KR, Golightly YM. Physical exercise as non-pharmacological treatment of chronic pain: why and when. Best Pract. Res. Clin. Rheumatol. 29(1), 120-130 (2015).

8 Stephens S, Feldman BM, Bradley N et al. Feasibility and effectiveness of an aerobic exercise program in children with fibromyalgia: results of a randomized controlled pilot trial. Arthritis Rheum. 59(10), 1399-1406 (2008).

9 Gowans SE, Dehueck A. Effectiveness of exercise in management of fibromyalgia. Curr. Opin. Rheumatol. 16(2), 138-142 (2004).

10 Williams DM, Dunsiger S, Emerson JA, Gwaltney CJ, Monti PM, Miranda R Jr. Self-paced exercise, affective response, and exercise adherence: a preliminary investigation using ecological momentary assessment. J. Sport Exerc. Psychol. 38(3), 282-291 (2016).

11 Koltyn KF, Brellenthin AG, Cook DB, Sehgal N, Hillard C. Mechanisms of exerciseinduced hypoalgesia. J. Pain. 15(12), 1294-1304 (2014).

12 Meeus M, Nijs J. Central sensitization: a biopsychosocial explanation for chronic widespread pain in patients with fibromyalgia and chronic fatigue syndrome. Clin. Rheumatol. 26(4), 465-473 (2007).

13 Lannersten L, Kosek E. Dysfunction of endogenous pain inhibition during exercise with painful muscles in patients with shoulder myalgia and fibromyalgia. Pain 151(1), 77-86 (2010).

14 Ellingson LD, Stegner AJ, Schwabacher IJ, Koltyn KF, Cook DB. Exercise strengthens central nervous system modulation of pain in fibromyalgia. Brain Sci. 6(1), 8 (2016).

15 Simons LE, Kaczynski KJ. The Fear Avoidance model of chronic pain: examination for pediatric application. J. Pain 13(9), 827-835 (2012).
16 Tran ST, Guite JW, Pantaleao A et al. Preliminary outcomes of a cross-site cognitive-behavioral and neuromuscular integrative training intervention for juvenile fibromyalgia. Arthritis Care Res.doi:10.1002/ acr.22946 (2016) (Epub ahead of print).

17 Thomas SM, Sil S, Kashikar-Zuck S, Myer GD. Can modified neuromuscular training support the treatment of chronic pain in adolescents? Strength Cond. J. 35(3), 12-26 (2013).

18 Kashikar-Zuck S, Tran ST, Barnett K et al. A qualitative examination of a new combined cognitive-behavioral and neuromuscular training intervention for juvenile fibromyalgia. Clin. J. Pain 32 (1), 70-81 (2015).

19 Kashikar-Zuck S, Ting TV, Arnold LM et al. Cognitive behavioral therapy for the treatment of juvenile fibromyalgia: a multisite, single-blind, randomized, controlled clinical trial. Arthritis Rheum. 64(1), 297-305 (2012).

20 Simons LE, Sieberg CB, Pielech M, Conroy C, Logan DE. What does it take? Comparing intensive rehabilitation to outpatient treatment for children with significant pain-related disability. J. Pediatr. Psychol. 38(2), 213-223 (2013).

21 Sherry DD, Brake L, Tress JL et al. The treatment of juvenile fibromyalgia with an intensive physical and psychosocial program. J. Pediatr. 167(3), 731-737 (2015). 